\title{
High-throughput sequencing in acute lymphoblastic leukemia: Follow-up of minimal residual disease and emergence of new clones
}

\author{
Mikaël Salson ${ }^{1 \star}$, Mathieu Giraud ${ }^{1 \star}$, Aurélie Caillault ${ }^{2 \star}$, Nathalie Grardel $^{2 \star}$, Nicolas Duployez $^{2}$, \\ Yann Ferret ${ }^{2}$, Marc Duez ${ }^{3}$, Ryan Herbert ${ }^{1}$, Tatiana Rocher ${ }^{1}$, Shéhérazade Sebda ${ }^{4}$, \\ Sabine Quief ${ }^{4}$, Céline Villenet ${ }^{4}$, Martin Figeac ${ }^{4,5}$, Claude Preudhomme ${ }^{2}$
}

Final accepted manuscript, as published in Leukemia Research 53 (2017) 1-7

http://dx.doi.org/10.1016/j.leukres.2016.11.009

प

\section{Highlights}

- Repertoire Sequencing (RepSeq) gives a deep insight of leukemic lymphoid populations.

- High-throughput sequencing identifies new emerging clones at the time of relapse.

- Clones of low abundance at diagnosis may become relapsing clones.

- The Vidjil software enables both automated and interactive analysis of RepSeq data.

\footnotetext{
${ }^{1}$ CRIStAL UMR 9189 CNRS, Université de Lille and Inria Lille, Lille, France; ${ }^{2}$ CHU Lille, Department of Hematology, Université de Lille, France; ${ }^{3}$ School of Social and Community Medicine, University of Bristol, Bristol, UK; ${ }^{4}$ Univ. Lille, plateforme de génomique fonctionnelle et structurale, F-59000 Lille, France; ${ }^{5} \mathrm{CHU}$ Lille, Cellule bioinformatique, plateau commun de séquençage, F-59000 Lille, France

* These authors contributed equally.

Co-corresponding authors: contact@vidjil.org (M. Salson and M. Giraud), aurelie.caillault@chru-lille.fr (A. Caillault), nathalie.grardel@chru-lille.fr (N. Grardel), claude.preudhomme@chru-lille.fr (C. Preudhomme).

Conflict of interest: None to report.
}

Cet article est diffusé librement selon les dispositions de l'article L533-4 du Code de la Recherche créé par la loi pour une République Numérique du 7 octobre 2016. This publication is freely distributed according to the French law.

\section{Abstract}

Minimal residual disease (MRD) is known to be an independent prognostic factor in patients with acute lymphoblastic leukemia (ALL). High-throughput sequencing (HTS) is currently used in routine practice for the diagnosis and follow-up of patients with hematological neoplasms. In this retrospective study, we examined the role of immunoglobulin/T-cell receptor-based MRD in patients with ALL by HTS analysis of immunoglobulin $H$ and/or T-cell receptor gamma chain loci in bone marrow samples from 11 patients with ALL, at diagnosis and during follow-up. We assessed the clinical feasibility of using combined HTS and bioinformatics analysis with interactive visualization using Vidjil software. We discuss the advantages and drawbacks of HTS for monitoring MRD. HTS gives a more complete insight of the leukemic population than conventional real-time quantitative PCR ( $q P C R)$, and allows identification of new emerging clones at each time point of the monitoring. Thus, HTS monitoring of Ig/TCR based MRD is expected to improve the management of patients with ALL.

Keywords: Acute Lymphoblastic Leukemia, Minimal Residual Disease, Follow-up, Repertoire Sequencing, Bioinformatics 


\section{Introduction}

\subsection{Assessment of Minimal Residual Disease in ALL}

Minimal residual disease (MRD) monitoring has proven to be one of the strongest independent prognostic factors in patients with acute lymphoblastic leukemia (ALL) [1, 2]. Sequential monitoring of MRD using sensitive and specific methods, such as quantitative real-time polymerase chain reaction (qPCR) or flow cytometry, has improved the assessment of treatment response [3, 4, 5] and is currently used for therapeutic stratification and the early detection of relapses [6, 7]. These methods allow the detection of a single leukemic cell among many normal cells.

MRD monitoring based on immunoglobulin (Ig) and T-cell receptor (TCR) gene recombinations has been standardized, and has become the gold standard method in routine practice [3, 4]. Ig and TCR recombinations occur in the early stages of B-cell and T-cell development. As a consequence, each lymphoid cell contains unique $\mathrm{V}(\mathrm{D}) \mathrm{J}$ recombinations resulting from random coupling between one of many possible $\mathrm{V}$, (D) and J genes (combinatorial diversity), as well as imprecise joining of gene segments and the addition of nucleotides to the DNA sequence at splice sites (junctional diversity) [8]. Identical recombinations thus reflect the clonal nature of a population, rather than being derived from independent cells.

Monitoring of Ig/TCR-based MRD in ALL consists of step-by-step analysis of $\mathrm{V}(\mathrm{D}) \mathrm{J}$ DNA recombinations in lymphoblasts, and their subsequent detection, with very high sensitivity, during follow-up. Leukemic clonal recombinations can be amplified by PCR and examined by capillary electrophoresis, and can then be isolated by polyacrylamide gel electrophoresis and finally sequenced by direct Sanger sequencing. Overall, clonal V(D)J recombinations occur in $>95 \%$ of ALL cases. Allele-specific oligonucleotides (ASOs) are designed to quantify MRD in follow-up samples by qPCR. However, this approach is time consuming in routine practice, and monitoring is therefore limited to the major leukemic clones (usually 1-3 markers per patient). This strategy can therefore yield false-negative results.

Failures of this MRD-monitoring strategy can occur as a result of absence of the initial marker, problems with the design of the customized primer sets (for ASO-PCR), clonal evolution during the course of the disease, or the emergence of a new clone at the time of relapse [9].

\subsection{Next-generation MRD Monitoring by High- Throughput Sequencing}

Repertoire sequencing (RepSeq) involves detailed sequencing of a lymphoid population, focusing on the V(D)J recombinations [10]. Several recent studies focused on MRD quantification by high-throughput sequencing (HTS) in patients with acute and chronic lymphoid disorders [11, 12]. Overall, HTS revealed higher sensitivity and precision in bone marrow and peripheral blood samples compared with multi-parameter flow cytometry or ASO-PCR. Notably, low MRD positivity by HTS was found in samples that were MRD-negative by standard methods [12]. In patients with ALL, MRD monitoring by HTS could predict the risk of relapse in both childhood [12, 13] and adult ALL [14], and both pre- and post-allogeneic stem cell transplantation [15, 16]. One of the benefits of the high sensitivity of HTS was the possibility of detecting MRD in peripheral blood, instead of bone marrow [15]. The use of HTS for MRD quantification may also allow the molecular heterogeneity of the lymphoid repertoire to be investigated. Interestingly, Kotrova et al. showed that patients with lower immunoglobulin heavy chain (IgH) diversity at day 78 had significantly lower relapse-free survival rates [13].

\subsection{Challenges}

HTS is a valuable technique at the time of ALL diagnosis, and allows the sequencing of clonal recombinations of multiple Ig/TCR genes by pooling several PCR systems in one experiment. It is therefore possible to identify leukemia-specific sequences from one or more clones in one or more PCR systems in a single step, without the need for Sanger sequencing [17].

HTS is an accurate, reliable, fast and relatively affordable technology, applicable to many routine 
practices. It is expected to improve MRD monitoring and enable the detection of multiple clones and subclones simultaneously, faster and with higher sensitivity than standard methods based on V(D)J sequencing. The use of HTS is also expected to reduce the failure rate of MRD quantification associated with clonal evolution or the emergence of new clones [18, 19, 20].

ASO-PCR is a standardized technique that usually focuses on 1-3 clones, while HTS is an emerging field, able to evaluate millions of sequences in a single experiment. The study by van Dongen et al. [1] recently listed the key challenges for high-throughput MRD technologies as broad availability, easy implementation, applicability in most patients, adequate sensitivity, fast, affordable, and standardized, with quality-assurance programs. It is essential to establish standardized protocols to limit variability among laboratories. These protocols should include the use of primer kits designed for multi-system PCR.

The quantity of data generated also raises several challenges. From a practical point of view, laboratories must increase their capacities to store and process the terabytes of data generated each year. Finally, it is also necessary to develop software for processing the data and synthetizing the results in an easily understandable way for its transfer into clinical practice.

\subsection{Repertoire sequencing (RepSeq) analysis software}

The high-throughput analysis of B or T cell receptor DNA sequences cannot be achieved using regular mapping software. This is because of the specificity of the problem, with a read usually containing a recombination between three genes with dozens of potential insertions and deletions [21]. These recombinations, non-templated insertions, deletions, and somatic hypermutations need to be integrated into the design of the algorithm.

RepSeq software usually performs optimized comparisons of HTS reads against a germline database. The international ImMunoGeneTics information system (IMGTR, http: / imgt .org/) has developed several tools for the in-depth analysis of $\mathrm{V}(\mathrm{D}) \mathrm{J}$ recombinations [22, 23, 24, 25]. New software to deal with HTS data (i.e., millions of sequences) has recently been developed, including [26], IgBlast [27], Decombinator [28], miTCR [29], Vidjil [30], TCRKlass [31], miXCR [32], and IMSEQ [33]. Several of these software tools cluster HTS reads into clones allowing relative quantification. However few of them offer interactive analysis features. The Vidjil open-source platform enables an autonomous usage in an immunology or hematology lab, from raw sequence files to analysis, annotation and storage [34].

\section{Material and Methods}

We conducted HTS-based MRD monitoring of IGH and/or T-cell receptor gamma chain (TRG) loci in patients with ALL. Data were analyzed using the Vidjil web application [30], which is directly usable by hematologists without the need for any dedicated bioinformatics support. This approach allowed us to identify new emerging clones at the time of relapse that were different from the main clones highlighted at diagnosis. We also assessed the reproducibility of our approach and compared our results with conventional ASO-PCR.

\subsection{Patient selection}

The clonality of 11 pediatric patients (8 B-ALL / 3 T-ALL, 5 female / 6 male, 3-19 years) was studied retrospectively in 43 bone-marrow samples (11 diagnosis and 32 follow-up time points, see Supplementary Material, Table 11. Approval for this study was obtained from the Institutional Review Board of CHRU of Lille (CSTMT093) and informed consent was obtained from the patients in accordance with the Declaration of Helsinki. Written informed consent was obtained from each patient or their parent or legal guardian.

\subsection{DNA extraction, library preparation and HTS}

We sequenced TRG (VGf1-10) and/or IGH by HTS in 11 patients with ALL at diagnosis and at several follow-up points. For every sample, $5 \times 10^{6}$ cells were extracted from bone marrow or blood using a QIAamp® DNA Mini Kit (Qiagen). DNA 
was measured using a Nanodrop system $\AA$, and $500 \mathrm{ng}$ were amplified by PCR with non-fluorescent BIOMED-2 TRG and/or IGH primers (Supplementary Material, Table 2). A total of 58 (11 diagnosis and 44 follow-up) samples were analyzed.

The samples were initially purified with MinElute $\mathbb{R}$ PCR Purification Kit (Qiagen). Libraries were prepared using an Ion Xpress ${ }^{\mathrm{TM}}$ Plus gDNA and Amplicon Library Kit (Life Technologies). Each library was barcoded using the Ion Xpress ${ }^{\mathrm{TM}}$ Barcode Adapters 1-96 Kit. The concentration of each barcoded library was controlled using an Agilent 2100 Bioanalyzer (Agilent Technologies), and the libraries were then pooled and their concentrations checked again with the Agilent 2100 Bioanalyzer.

The libraries were amplified by emulsion PCR using an Ion OneTouch ${ }^{\mathrm{TM}} 2$ (Life Technologies) with an Ion PGM ${ }^{\mathrm{TM}}$ Template OT2 400 Kit (Life Technologies). After amplification, the libraries were enriched using an Ion OneTouch ${ }^{\mathrm{TM}}$ ES (Life Technologies). The PCR products were verified using an Ion Sphere ${ }^{\mathrm{TM}}$ Quality Control Kit With a Qubit $\AA$ 2.0 Fluorometer (Invitrogen). The libraries were sequenced using an Ion Personal Genome Machine (Ion PGM ${ }^{\mathrm{TM}}$ ) with an Ion PGM ${ }^{\mathrm{TM}}$ Sequencing 400 Kit and Ion $318^{\mathrm{TM}}$ Chip Kit (Life Technologies).

\subsection{Bioinformatics analysis and visualization}

The raw Ion Torrent flow was transformed to demultiplexed FastQ sequences using the Torrent Server from Life Technologies, allowing 0 error in barcode splitting.

The reads were processed using Vidjil software (www.vidjil.org, version 2015.01), with parameters to search for multiple loci and incomplete recombinations and to avoid spurious hits $(-i-e \quad 1-z$ $100-r \quad 1-3-d)$. Vidjil gathers the reads into clones on the basis of their $\mathrm{V}(\mathrm{D}) \mathrm{J}$ recombinations. Two reads belong to the same clone if they share the same "window" overlapping the actual CDR3. This $50 \mathrm{bp}$ window is large enough to be highly specific. Window detection is based on a fast bioinformatics method using $k$-mers indexing of germline genes [30].

We examined the lymphocyte populations of the 11 patients using the Vidjil web application, tracking the clones over time. Interactive visualization of these populations can be accessed at http://www.vidjil.org/data/\#2016-lr. Vidjil outputs provided a representative sequence for each of the most-represented clones. All fragments of the representative sequence are guaranteed to be shared by at least $50 \%$ of the reads assigned to the clone. V(D)J recombination analysis (determination of $\mathrm{V}(\mathrm{D}) \mathrm{J}$ germline genes and $\mathrm{N}$ region) was only performed at the end of the process.

Technical errors in PCR and sequencing, as well as somatic mutations, mean that several clones with slight variations may be detected. A manual review was performed in the web application, and, when the pattern clearly indicated a sequencing error, clones were merged.

\section{Results}

Patient follow-up requires the quantification of clones even at very low concentrations. The detection level depends directly on the depth of the sequencing (ie. the number of reads obtained). On average we obtained 1.2 million reads per sample (median: 0.98 million) of which $74 \%$ could be analyzed by Vidjil (median: 77\%). Figure 11 shows the evolution of the main clones for those patients analyzed by the Vidjil web application. We discuss the potential clinical applications of this multiclonal analysis by detailing four patient cases.

Patient 010 (TRG). This patient with T-ALL had one relapse at D413 (after diagnosis) and underwent allogeneic transplantation at D486. The two last time points (D445 and D475) were after two NECTAR (nelarabine, etoposide and cyclophosphamide) cycles, before allogeneic transplantation. The main clone was undetectable by conventional technique initially (D81) and post-NECTAR, but was detected by HTS even at a very low level (4 and 11 reads). The patient remained relapse-free 2 years after transplantation.

Patient 013 (IGH). This patient with pre-B (BIII) ALL was treated in the standard-risk (AR1) group of the EORTC-58081 protocol. The sequence of the main clone of IGH was not identified by conventional technique, while HTS analysis showed a con- 
010 (TRG)

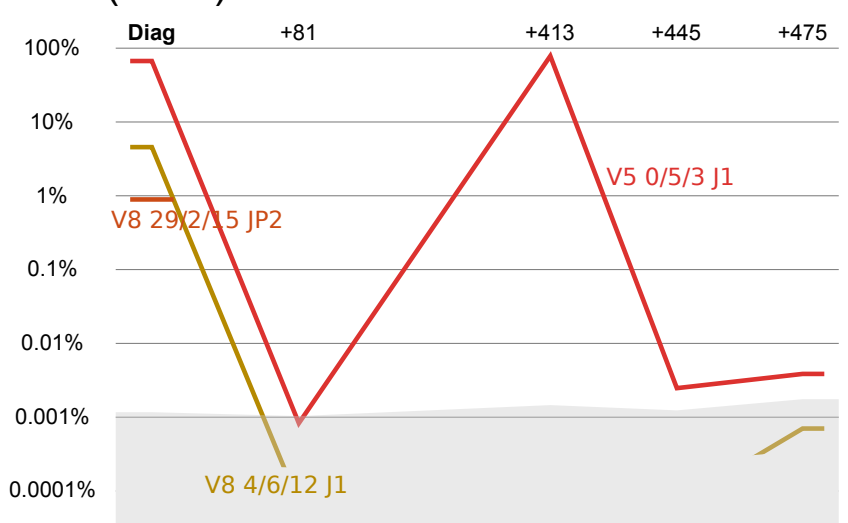

063 (TRG)

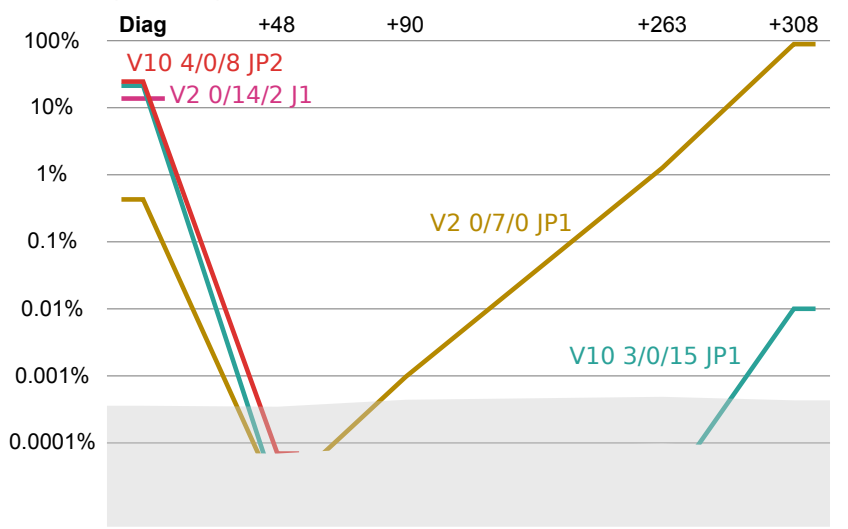

\section{4 (TRG)}

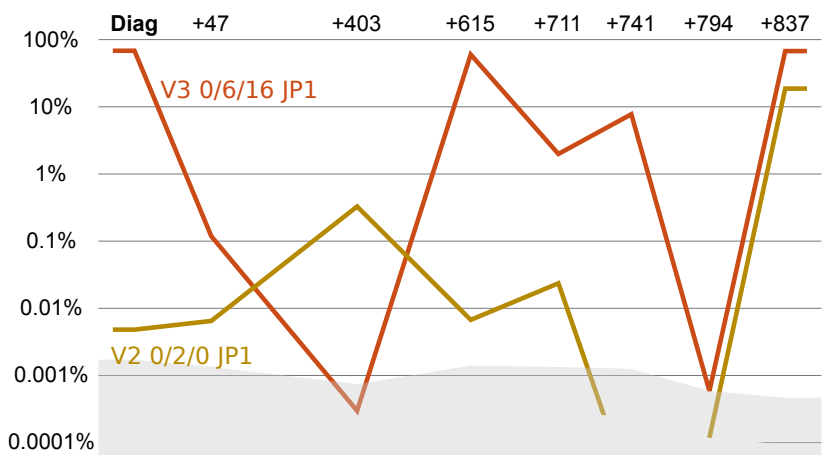

$013(\mathrm{IGH})$

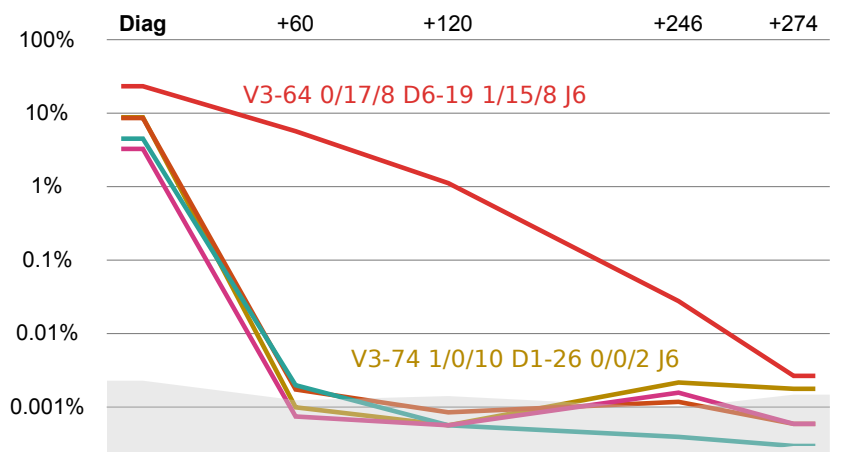

$0.0001 \%$

\section{$063(\mathrm{IGH})$}

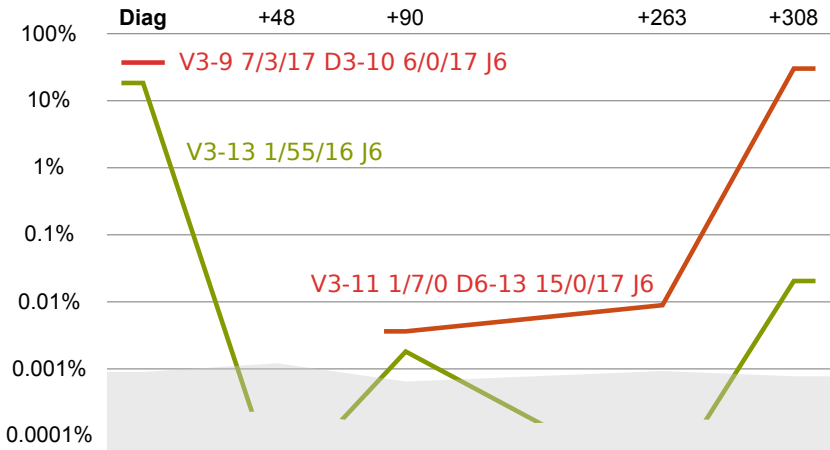

\section{$064(\mathrm{IGH})$}

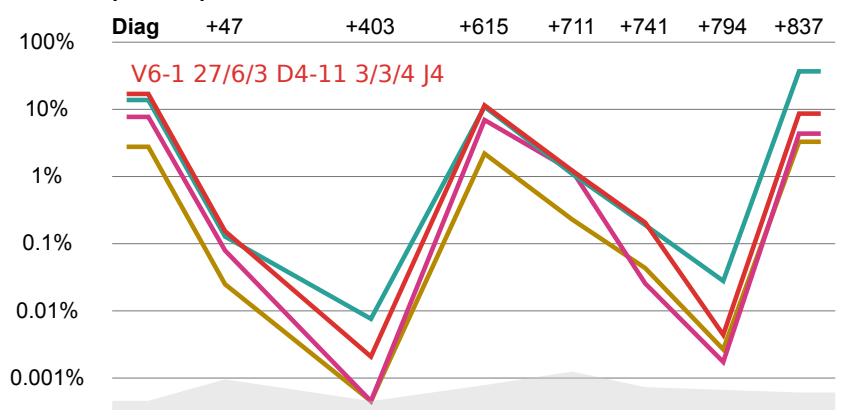

$0.0001 \%$

Figure 1: Top: Follow-up of patient 010 for TRG (left), and of patient 013 for IGH (right). Middle and bottom: Follow-up of patients 063 and 064, both for TRG (left) and IGH (right). The $x$-axis shows the days post-diagnosis, and the $y$-axis the concentration of the clones. All the curves were obtained after manual inspection and additional clustering of the clones. Red lines are the clones followed by ASO-PCR, and the background clones are hidden. The complete interactive curves for the 11 patients, and the nucleic sequences of the clones and their $\mathrm{V}(\mathrm{D}) \mathrm{J}$ designations, can be accessed at http://www.vidjil.org/data/\#2016-lr. 
tinuous decrease in the concentration of the main clone. However, the patient failed to respond to EORTC-58081 induction, and was switched to the high-risk (VHR) therapy arm, and allogeneic transplantation was planned for D315. The two main clones at diagnosis both showed decreasing profiles, but at different rates (see Figure 11Patient 013).

Patient 063 (TRG+IGH). This patient with BII ALL received a stem cell transplantation at day D183. The clone identified at diagnosis and followed by ASO-PCR, TRGV10*01 -4/0/-8 TRGJP2*01, was undetectable after transplantation. However, the patient relapsed with another clone that was not identified at diagnosis by conventional technique. Interestingly the relapse clone (D308) of TRG, TRGV2*01 -0/7/-0 TRGJP1*01, was already observable by HTS at day D90, and was the main clone at D263 (1.3\% of the reads). This TRG clone was present at diagnosis, but at a concentration two orders of magnitude below that of the main clone. However other clones maintained a steady concentration throughout, while this clone increased in concentration. There was no similar IGH clone: the main IGH clones at D308 were not detected at diagnosis, even with HTS. The raw sequence files for this patient can be accessed in the Supplementary Material.

Patient 064 (TRG+IGH). This patient with BII ALL and complex karyotypic abnormalities had two relapses at days D615 and D837, respectively. The main TRG (TRGV3*01 -0/6/-16 TRGJP1*01) and IGH clones detected (IGHV6-1*01 -27/19/-4 IGHJ ${ }^{*} 02$ ) showed parallel evolution. These sequences therefore probably came from the same clonal population. At the second relapse, at D837, another TRG clone accounted for about $20 \%$ of the reads (TRGV2*02 -0/2/-0 TRGJP1*01). This clone was present at diagnosis and showed noticeable growth even at D403. No IGH clone showed similar evolution. Unfortunately, this patient died 4 years after the diagnosis.

The seven other patients show a more simple clonal evolution. Their cases are described in Supplementary Material.

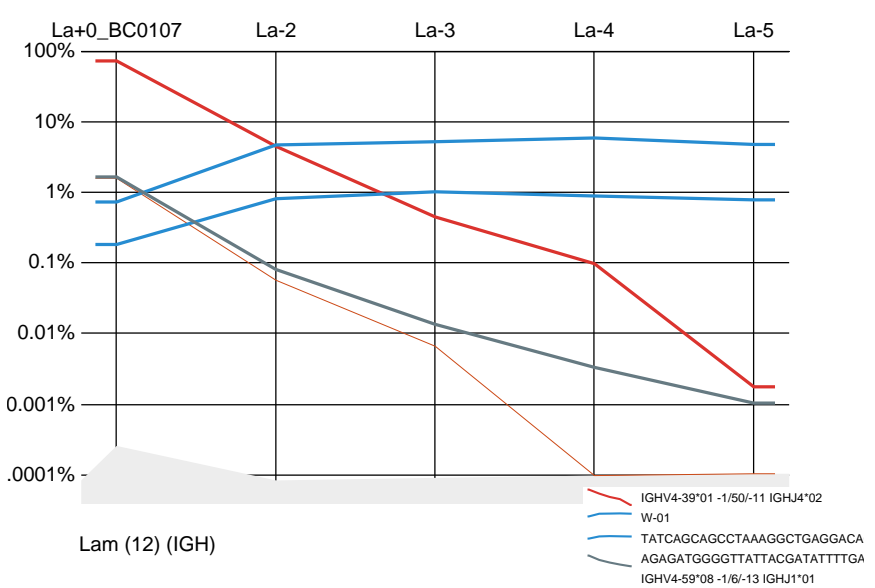

Figure 2: Dilution scale, for diagnosis of patient 012 (IGH). Horizontal lines are clones from the spiked-in controls.

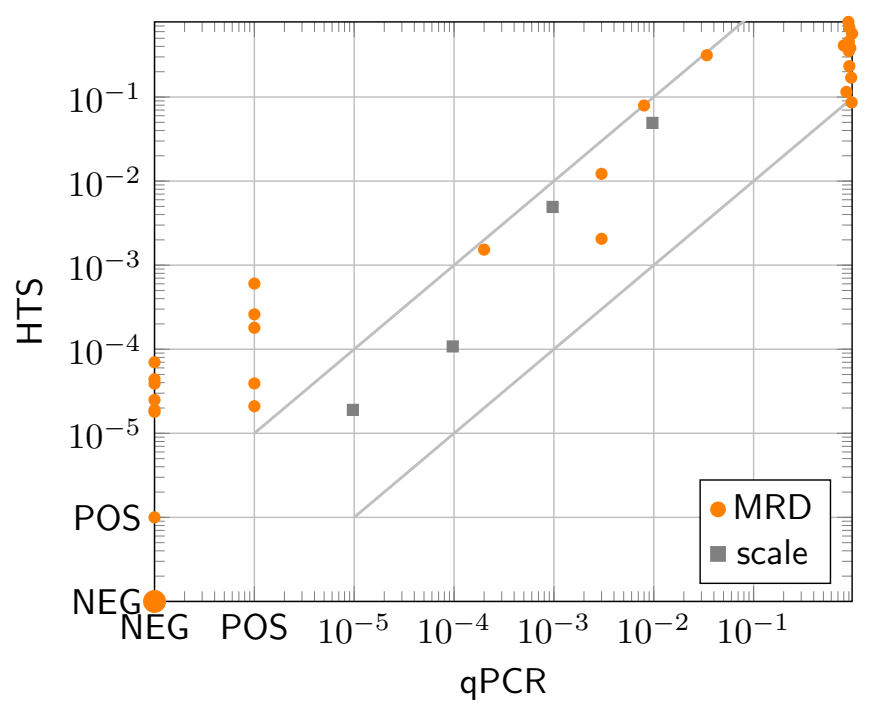

Figure 3: Comparison between clone ratios detected by HTS analyzed by Vidjil and qPCR ratios (circles, 32 samples) and theoretical ratios for the scale (boxes, 4 samples, see Figure 2). HTS ratios are of numbers of reads, without additional clustering to account for potential sequencing errors. The NEG-NEG point is large because four samples were negative in both qPCR and HTS. 


\subsection{Quantification scale}

To assess the quality of the quantification, it is essential to understand the limits of the technology. A diagnostic sample was diluted with one pooled peripheral blood lymphocyte sample from five healthy donors, to yield concentrations of $1 \%$, $0.1 \% 0.01 \%$ and $0.001 \%$ of the initial concentration at diagnosis, respectively. Two sequences for quantification control were also added to each sample for quality assessment. We added two previously categorized diagnosis samples to each sample before PCR, to generate two consistent markers along the scale. Consistent marker samples were added to represent $1 \%$ and $0.1 \%$ of the total DNA, respectively.

As expected, the concentration of the main clone decreased by about one tenth for each dilution (Figures 2 and 3). At a dilution of $10^{-2}$, the main clone had a concentration of 0.05 compared with that at diagnosis, at $10^{-3}$ it was 0.005 , at $10^{-4}$ it was 0.001 , and at $10^{-5}$ it was 0.00002 . The exact resolution depended on the depth of the sequencing. This depth should be set depending on the application (few reads are required to identify major clones at diagnosis, while more are needed to determine the concentration of clones of interest during follow-up).

\subsection{Reproducibility}

Comparison between HTS and qPCR. HTS and qPCR do not measure the same parameters. For HTS, the ratios reported by Vidjil take the number of analyzed reads as a reference, which ideally corresponds to the number of rearranged $\mathrm{T}$ or $\mathrm{B}$ cells in the studied system. In contrast, qPCR measures the proportion of total cells, which also includes other mononucleic cells.

We compared the results for qPCR and HTS in 32 samples (Figure 3). The results for 22 samples were within one log of each other by both techniques. HTS detected reads in five samples where the standard technique detected nothing, including two samples from patient 010 discussed above. All these clones were detected below the $10^{-4}$ ratio. The inclusion of a standard of known concentration could be used to calibrate these different ratios. Care should be taken to avoid contamination between samples, e.g., by handling and sequencing diagnosis and follow-up samples from the same patient separately, as in routine practice.

Reproducibilities of PCR and HTS. We assessed the reproducibility of PCR and HTS by duplicating the initial PCR step (PCR A and PCR B). We also took a sample from PCR A and labelled it with two different barcodes. This allowed us to assess which step was more reproducible. The results for patient 014 are shown in Figure 4. The reproducibility was generally good, except at very low concentrations $\left(<10^{-4}\right)$. The spread of distribution at lower concentrations comes in part from PCR and HTS biases, but also from biological sampling.

However, in PCR duplicates, clones at a concentration greater than $10^{-2}$ were detected in one situation, but not at all in another. We examined the sequences in those cases in detail (Figure 2), and compared them to the closest clone. The results suggested that the misidentification may have been largely caused by errors in the sequencing or PCR process (see Supplementary Material).

\section{Discusssion}

Faham et al [12] have shown what high-throughput sequencing can bring to monitor the leukemic clones along the time by trying to reproduce the measurements that were made using qPCR. Accordingly to this article showing that both techniques are well correlated, we were only interested to study the contribution of HTS to patient monitoring and multi-clonality evolution. Indeed, HTS also offers a new opportunity: looking at the evolution of all the clones. We have shown that several distinct clones can have high concentrations at diagnosis. With HTS it is now possible to follow their concentration. More importantly, as relapse can occur with clones of low abundance at diagnosis, HTS could help identifying such relapse ahead.

Another gret interest in HTS has been emphasized by Kotrová et al [13]. They have identified a possible new stratification method relying on clonal diversity, a measure that can only be obtained using HTS. 
The purpose of this study is to show what highthroughput sequencing could bring compared to ASO-PCR. Other studies have shown what ASOPCR brings [35, 36]. Comparison of HTS with other methods should be addressed by future studies.

\subsection{Management of sequencing errors.}

We have shown that some, albeit rare, cases of sequencing/PCR errors emphasize the need for highfidelity PCR enzymes, which would reduce the risk of misleading errors in the quantification of MRD [37]. Moreover, users should indeed be aware of the type of errors that the sequencer may produce [38].

Sequences harboring PCR or sequencing errors can be clustered manually or automatically using interactive software such as the Vidjil web application [17]. However in some cases, it is difficult to determine if differences are the result of sequencing or PCR errors, recombinations, or further somatic mutations. For example, see Supplementary Material.

\subsection{Conclusion}

HTS provides new opportunities, both for MRD monitoring and for furthering our knowledge of lymphoid pathologies. Coupled with interactive bioinformatics analysis, HTS gives a more complete insight into the blastic population at diagnosis, and allows observation of the evolution of this population, as well as emerging new ones. The development of standardized protocols with multi-system PCR and calibration standards could help in developing these techniques.

\section{References}

[1] Jacques JM van Dongen, Vincent HJ van der Velden, Monika Brüggemann, and Alberto Orfao. Minimal residual disease (MRD) diagnostics in acute lymphoblastic leukemia (ALL): need for sensitive, fast and standardized technologies. Blood, 125(26):3996-4009, 2015.
[2] Monika Brüggemann, Thorsten Raff, and Michael Kneba. Has MRD monitoring superseded other prognostic factors in adult ALL? Blood, 120(23):4470-4481, 2012.

[3] J. J. M. van Dongen, T. Szczepański, and H. J. Adriaansen. Immunobiology of leukemia. In Henderson ES, Lister TA, and Greaves M, editors, Leukemia, pages 85-130. WB Saunders, Philadelphia, 7th edition, 2002.

[4] J. J. M. van Dongen, A. W. Langerak, M. Brüggemann, P. A. S. Evans, M. Hummel, F. L. Lavender, E. Delabesse, F. Davi, E. Schuuring, R. García-Sanz, J. H. J. M. van Krieken, J. Droese, D. González, C. Bastard, H. E. White, M. Spaargaren, M. González, A. Parreira, J. L. Smith, G. J. Morgan, M. Kneba, and E. A. Macintyre. Design and standardization of PCR primers and protocols for detection of clonal immunoglobulin and T-cell receptor gene recombinations in suspect lymphoproliferations: report of the BIOMED-2 concerted action BMH4-CT98-3936. Leukemia, 17(12):2257-317, 2003.

[5] T. Kalina, J. Flores-Montero, V. H. J. van der Velden, M. Martin-Ayuso, S. Bottcher, M. Ritgen, J. Almeida, L. Lhermitte, V. Asnafi, A. Mendonca, R. de Tute, M. Cullen, L. Sedek, M. B. Vidriales, J. J. Perez, J. G. te Marvelde, E. Mejstrikova, O. Hrusak, T. Szczepanski, J. J. M. van Dongen, and A. Orfao. Euroflow standardization of flow cytometer instrument settings and immunophenotyping protocols. Leukemia, 26(9):1986-2010, 2012.

[6] Hélène Cavé, Jutte van der Werff Ten Bosch, Stefan Suciu, Christine Guidal, Christine Waterkeyn, Jacques Otten, Marleen Bakkus, Kris Thielemans, Bernard Grandchamp, Etienne Vilmer, Brigitte Nelken, Martine Fournier, Patrick Boutard, Emmanuel Lebrun, Françoise Méchinaud, Richard Garand, Alain Robert, Nicole Dastugue, Emmanuel Plouvier, Evelyne Racadot, Alice Ferster, Jan Gyselinck, Odile Fenneteau, Michel Duval, Gabriel Solbu, and Anne-Marie Manel. Clinical significance of minimal residual disease in childhood acute 
lymphoblastic leukemia. New England Journal of Medicine, 339(9):591-598, 1998.

[7] Virginie Gandemer, Cécile Pochon, Emmanuel Oger, Jean-Hugues H Dalle, Gérard Michel, Claudine Schmitt, Eva Berranger, Claire Galambrun, Hélène Cavé, Jean-Michel Cayuela, Nathalie Grardel, Elizabeth Macintyre, Geneviève Margueritte, Françoise Méchinaud, Pierre Rorhlich, Patrick Lutz, François Demeocq, Pascale Schneider, Dominique Plantaz, Marilyne Poirée, and Pierre Bordigoni. Clinical value of pre-transplant minimal residual disease in childhood lymphoblastic leukaemia: the results of the French minimal residual disease-guided protocol. British Journal of Haematology, 165(3):392-401, 2014.

[8] Susumu Tonegawa. Somatic generation of antibody diversity. Nature, 302(5909):575-581, 1983.

[9] Gunter Kerst, Hermann Kreyenberg, Carmen Roth, Catrin Well, Klaus Dietz, Elaine Coustan-Smith, Dario Campana, Ewa Koscielniak, Charlotte Niemeyer, Paul G. Schlegel, Ingo Müller, Dietrich Niethammer, and Peter Bader. Concurrent detection of minimal residual disease (MRD) in childhood acute lymphoblastic leukaemia by flow cytometry and real-time PCR. British Journal of Haematology, 128(6):774-782, 2005.

[10] William H. Robinson. Sequencing the functional antibody repertoire - diagnostic and therapeutic discovery. Nature Reviews Rheumatology, 11(3):171-182, 2015.

[11] Aaron C. Logan, Hong Gao, Chunlin Wang, Bita Sahaf, Carol D. Jones, Eleanor L Marshall, Ismael Buno, Randall Armstrong, Andrew Z. Fire, Kenneth I Weinberg, Michael Mindrinos, James L. Zehnder, Scott D. Boyd, Wenzhong Xiao, Ronald W. Davis, and David B. Miklos. High-throughput VDJ sequencing for quantification of minimal residual disease in chronic lymphocytic leukemia and immune reconstitution assessment. Proceedings of the National
Academy of Sciences of the United States of America, 108(52):21194-21199, 2011.

[12] Malek Faham, Jianbiao Zheng, Martin Moorhead, Victoria E H Carlton, Patricia Stow, Elaine Coustan-Smith, Ching-Hon Pui, and Dario Campana. Deep-sequencing approach for minimal residual disease detection in acute lymphoblastic leukemia. Blood, 120(26):51735180, 2012.

[13] Michaela Kotrova, Katerina Muzikova, Ester Mejstrikova, Michaela Novakova, Violeta Bakardjieva-Mihaylova, Karel Fiser, Jan Stuchly, Mathieu Giraud, Mikaël Salson, Christiane Pott, Monika Brüggemann, Marc Füllgrabe, Jan Stary, Jan Trka, and Eva Fronkova. The predictive strength of next generation sequencing MRD detection for relapse compared with current methods in childhood ALL. Blood, 126(8):1045-1047, 2015.

[14] Olga Sala Torra, Megan Othus, David W Williamson, Brent L. Wood, Ilan Kirsch, Harlan Robins, Margaret O'Donnell, Stephen J. Forman, Frederick R. Appelbaum, and Jerald P Radich. Minimal Residual Disease detection by Next Generation Sequencing in adult B-cell Acute Lymphoblastic Leukemia (ALL) patients treated on SWOG trial S0333. Blood, 124(21):2399-2399, 2014.

[15] Aaron C. Logan, Nikita Vashi, Malek Faham, Victoria Carlton, Katherine Kong, Ismael Buno, Jianbiao Zheng, Martin Moorhead, Mark Klinger, Bing Zhang, Amna Waqar, James L. Zehnder, and David B. Miklos. Immunoglobulin and $\mathrm{T}$ cell receptor gene high-throughput sequencing quantifies minimal residual disease in acute lymphoblastic leukemia and predicts post-transplantation relapse and survival. Biology of Blood and Marrow Transplantation, 20(9):1307-1313, 2014.

[16] Michael A. Pulsipher, Chris Carlson, Bryan Langholz, Donna A. Wall, Kirk R. Schultz, Nancy Bunin, Ilan Kirsch, Julie M. GastierFoster, Michael Borowitz, Cindy Desmarais, David Williamson, Michael Kalos, and 
Stephan A. Grupp. IgH-V(D)J NGSMRD measurement pre- and early postallotransplant defines very low- and very high-risk ALL patients. Blood, 125(22):35013508, 2015.

[17] Yann Ferret, Aurélie Caillault, Shéhérazade Sebda, Marc Duez, Nathalie Grardel, Nicolas Duployez, Céline Villenet, Martin Figeac, Claude Preudhomme, Mikaël Salson, and Mathieu Giraud. Multi-loci diagnosis of acute lymphoblastic leukemia with highthroughput sequencing and bioinformatics analysis. British Journal of Haematology, 173(3):413-420, 2016.

[18] A.W. Langerak, M. Brüggemann, N. Darzentas, M. Catherwood, G. Cazzaniga, F. Davi, J.J.M van Dongen, P.A.S. Evans, R. Garcia Sanz, V. Giudicelli, D. Gonzalez, P.J.T.A. Groenen, M. Hummel, M.-P. Lefranc, E.A. Macintyre, C. Pott, and K. Stamatopoulos. Technical develoments: general principles and available technologies. In Second ESLHO Symposium, pages 11-19, 2013.

[19] Harlan Robins. Immunosequencing: applications of immune repertoire deep sequencing. Current Opinion in Immunology, 25, 2013.

[20] George Georgiou, Gregory C Ippolito, John Beausang, Christian E Busse, Hedda Wardemann, and Stephen R Quake. The promise and challenge of high-throughput sequencing of the antibody repertoire. Nature Biotechnology, 32:158-168, 2014.

[21] Jennifer Benichou, Rotem Ben-Hamo, Yoram Louzoun, and Sol Efroni. Rep-Seq: uncovering the immunological repertoire through next-generation sequencing. Immunology, 135(3):183-91, 2012.

[22] Mehdi Yousfi Monod, Véronique Giudicelli, Denys Chaume, and Marie-Paule Lefranc. IMGT/JunctionAnalysis: the first tool for the analysis of the immunoglobulin and $\mathrm{T}$ cell receptor complex V-J and V-D-J JUNCTIONs. Bioinformatics, 20(S1):i379-85, 2004.
[23] Xavier Brochet, Marie-Paule Lefranc, and Véronique Giudicelli. IMGT/V-QUEST: the highly customized and integrated system for IG and TR standardized V-J and V-D-J sequence analysis. Nucleic Acids Research, 36(S2):W503-W508, 2008.

[24] Marie-Paule Lefranc. IMGT, the International ImMunoGeneTics Information System. Cold Spring Harbor Protocols, 2011(6):pdb.top115, 2011.

[25] Eltaf Alamyar, Véronique Giudicelli, Shuo Li, Patrice Duroux, and Marie-Paule Lefranc. IMGT/HighV-QUEST: the IMGT $R$ web portal for immunoglobulin (IG) or antibody and $\mathrm{t}$ cell receptor (TR) analysis from NGS high throughput and deep sequencing. Immunome Research, 8(1), 2012.

[26] Ramy Arnaout, William Lee, Patrick Cahill, Tracey Honan, Todd Sparrow, Michael Weiand, Chad Nusbaum, Klaus Rajewsky, and Sergei B. Koralov. High-resolution description of antibody heavy-chain repertoires in humans. PLoS ONE, 6(8):e22365, 2011.

[27] Jian Ye, Ning Ma, Thomas L. Madden, and James M. Ostell. IgBLAST: an immunoglobulin variable domain sequence analysis tool. Nucleic Acids Research, 41:W34-W40, 2013.

[28] Niclas Thomas, James Heather, Wilfred Ndifon, John Shawe-Taylor, and Benjamin Chain. Decombinator: a tool for fast, efficient gene assignment in T-cell receptor sequences using a finite state machine. Bioinformatics, 29(5):542550, 2013.

[29] Dmitriy A Bolotin, Mikhail Shugay, Ilgar Z Mamedov, Maria A Turchaninova Ekaterina $\mathrm{V}$ Putintseva, Ivan V Zvyagin, Olga V Britanova, and Dmitriy M Chudakov. MiTCR: software for T-cell receptor sequencing data analysis. Nature Methods, 10:813-814, 2013.

[30] Mathieu Giraud, Mikaël Salson, Marc Duez, Céline Villenet, Sabine Quief, Aurélie Caillault, Nathalie Grardel, Christophe Roumier, Claude Preudhomme, and Martin Figeac. Fast 
multiclonal clusterization of $\mathrm{V}(\mathrm{D}) \mathrm{J}$ recombinations from high-throughput sequencing. BMC Genomics, 15(1):409, 2014.

[31] Xi Yang, Di Liu, Na Lv, Fangqing Zhao, Fei Liu, Jing Zou, Yan Chen, Xue Xiao, Jun Wu, Peipei Liu, Jing Gao, Yongfei Hu, Yi Shi, Jun Liu, Ruifen Zhang, Chen Chen, Juncai Ma, George F Gao, and Baoli Zhu. TCRklass: A new k-string-based algorithm for human and mouse TCR repertoire characterization. Journal of Immunology, 194(1), 2014.

[32] Dmitriy A. Bolotin, Stanislav Poslavsky, Igor Mitrophanov, Mikhail Shugay, Ilgar Z. Mamedov, Ekaterina V. Putintseva, and Dmitriy M. Chudakov. MiXCR: software for comprehensive adaptive immunity profiling. Nature Methods, 12(5):380-381, 2015.

[33] Leon Kuchenbecker, Mikalai Nienen, Jochen Hecht, Avidan U. Neumann, Nina Babel, Knut Reinert, and Peter N. Robinson. IMSEQ - a fast and error aware approach to immunogenetic sequence analysis. Bioinformatics, 31(18):btv309, 2015.

[34] Marc Duez, Mathieu Giraud, Ryan Herbert, Tatiana Rocher, Mikaël Salson, and Florian Thonier. Vidjil: A web platform for analysis of high-throughput repertoire sequencing. PLOS One, 11(11):e0166126, 2016.

[35] Kheira Beldjord, Sylvie Chevret, Vahid Asnafi, Françoise Huguet, Marie-Laure Boulland, Thibaut Leguay, Xavier Thomas, JeanMichel Cayuela, Nathalie Grardel, Yves Chalandon, et al. Oncogenetics and minimal residual disease are independent outcome predictors in adult patients with acute lymphoblastic leukemia. Blood, 123(24):3739-3749, 2014.

[36] R Garand, K Beldjord, H Cavé, C Fossat, I Arnoux, V Asnafi, Y Bertrand, ML Boulland, C Brouzes, E Clappier, et al. Flow cytometry and IG/TCR quantitative PCR for minimal residual disease quantitation in acute lymphoblastic leukemia: a French multicenter prospective study on behalf of the FRALLE, EORTC and GRAALL. Leukemia, 27(2):370376, 2013.
[37] Michael A. Quail, Thomas D. Otto, Yong Gu, Simon R. Harris, Thomas F. Skelly, Jacqueline A. McQuillan, Harold P. Swerdlow, and Samuel O. Oyola. Optimal enzymes for amplifying sequencing libraries. Nature Methods, 9(1):10-11, January 2012.

[38] Michael G. Ross, Carsten Russ, Maura Costello, Andrew Hollinger, Niall J. Lennon, Ryan Hegarty, Chad Nusbaum, and David B. Jaffe. Characterizing and measuring bias in sequence data. Genome Biology, 14(5):R51, 2013.

\section{Acknowledgements}

This work was supported by SIRIC ONCOLille [grant number INCa-DGOS-Inserm 6041]. We thank the anonymous reviewers for their constructive comments on this manuscript.

\section{Author contribution}

MS, MG, AC and NG were equal contributors. AC, $\mathrm{CP}$ and NG selected the patients. MF, SS, SQ, CV and $\mathrm{AC}$ designed the sequencing protocol. MD, MG, MS, RH and TR conceived the Vidjil software. MS, MG, AC, ND, NG, MF, SS and YF analyzed and discussed the data. MS, MG, ND and YF drafted the paper. All authors corrected the paper and approved the final manuscript. 

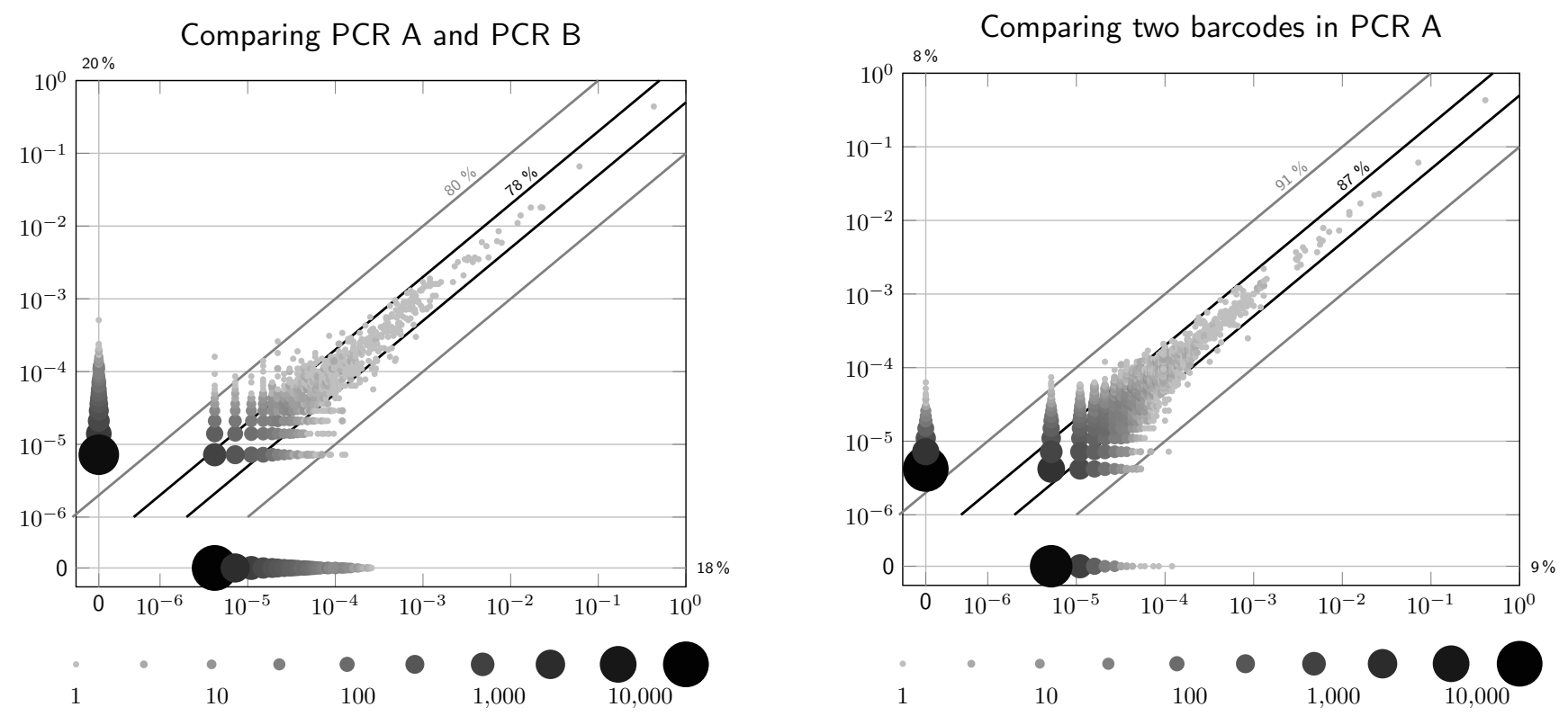

Comparing PCR A and PCR B

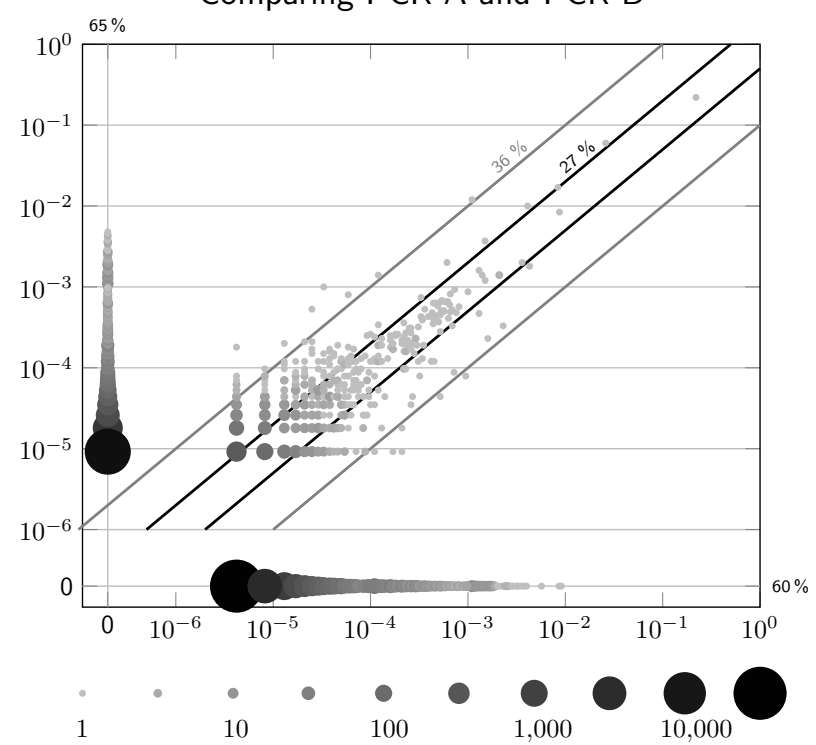

Comparing two barcodes in PCR A

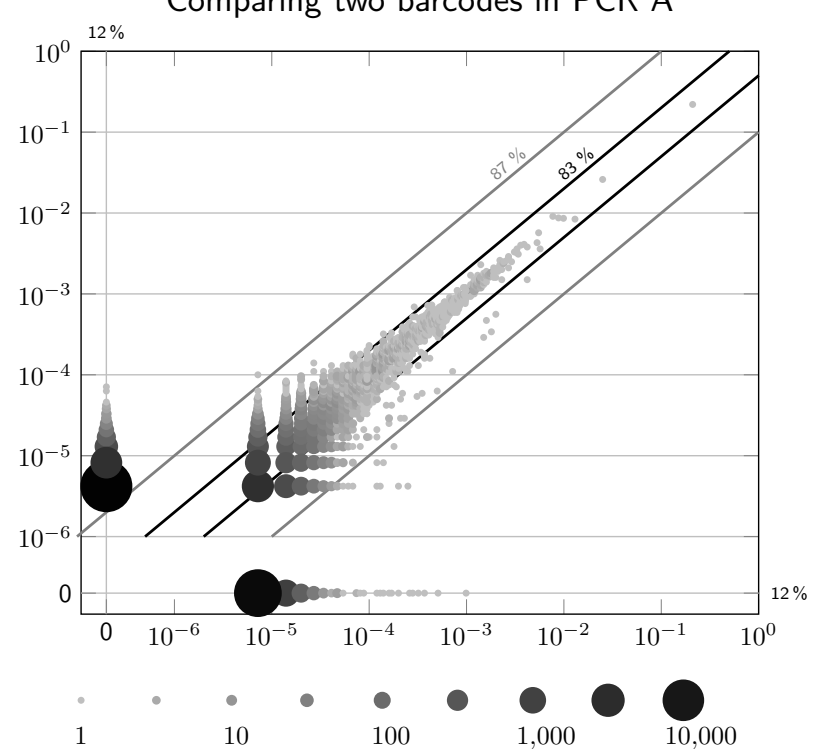

Comparing PCR A and PCR B
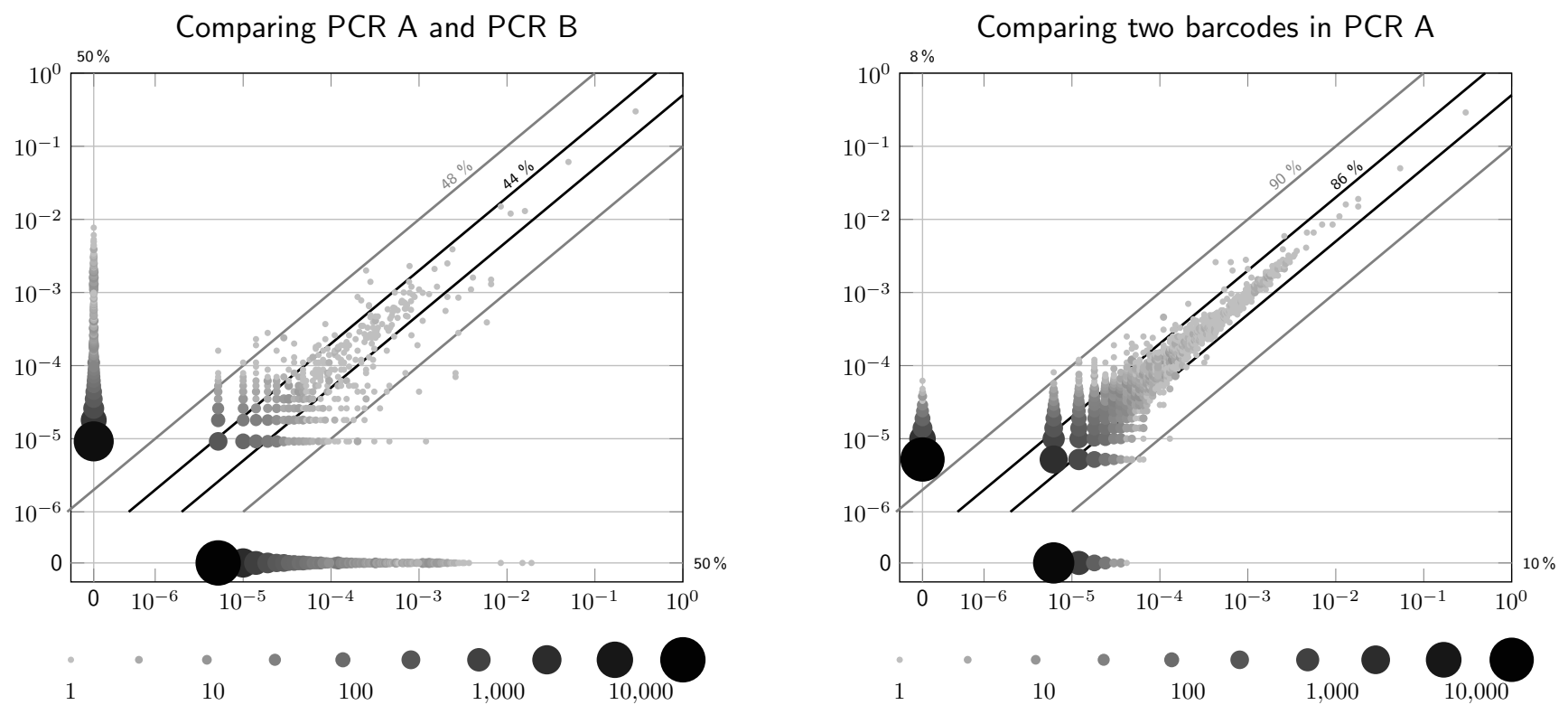

Figure 4: Reproducibility of PCR (left column) and sequencing (right column) in patient 014 at diagnosis (top), and at first (middle) and second follow-up points (bottom). Each point represents a set of clones identified by bioinformatics processing, before manual clustering. Ratios are numbers of reads for both experiments. The size of the point indicates the number of clones sharing the same value on both axes. 


\section{Supplementary Material}

\section{Raw data on the patient 063}

The raw sequence files (.fastq.gz) for the patient 063 (TRG+IGH, diagnosis + follow-ups at days D48, D90, D263 and D308) can be accessed at http://www.vidjil.org/data/\#2016-lr.

\section{Results on the seven other patients}

The interactive visualization of the clonal populations of the seven patients not described in the main text can be accessed at http://www.vidjil.org/data/\#2016-lr. In the following we denote by "main clone" the clone identified at diagnosis by conventional technique and then followed by ASO-PCR.

Patient 009 (TRG). The major clone is stable on the two follow-up points, above $1 \%$.

Patient 011 (TRG). Two clones have similar evolution. Their relative concentration do not evolve much from first follow-up (below $10^{-3}$ ).

Patient 012 (IGH). The main clone is below $10^{-4}$ in HTS (neg in qPCR) for the two follow-ups but it is still present.

Patient 014 (IGH). The main clone is above $10^{-4}$ at first follow-up (but positive below $10^{-4}$ with qPCR).

Patient 099 (TRG). Two different clones represent about $90 \%$ of the reads at diagnosis. They both decrease below $1 \%$ at D107.

Patient 100 (TRG+IGH). The major clone in IGH decreases below $10^{-3}$ at $\mathrm{D} 42$, and another major clone at diagnosis (25\%) completely disappears at D42. On TRG, all major clones identified at diagnosis disappear at the follow-up.

Patient 101 (IGH). The two major clones represent about $80 \%$ of the reads. Their concentration both drop below $10^{-4}$ at D44.

Note that the concentrations were not evaluated by qPCR for the three last patients as the Sanger sequencing failed in those cases.
Pitfalls in differentiating between technical bias and biological mutations

The four clones shown in Supplementary Material, Fig 3, all supported by more than 30,000 reads, only differed at two sites with homopolymeric mutations G / GGGG. However, it was not clear if these represented sequencing errors or biologically distinct clones. Given that Ion Torrent sequencers are prone to homopolymeric errors, and that the four curves showed parallel evolution (Supplementary Material, Fig 1), suggested that these were probably sequencing errors in specific sequences, which are a relatively common error with sequencers. However the follow-up points were sequenced in two independent runs several months apart. Moreover if these were sequencing errors, we would also expect to observe stretches of two and three Gs. It should also be noted that deleting three Gs, as observed in the sequences, did not induce a frame shift. For MRD follow-up, it may be interesting to follow the evolution of both the individual clones and the four sequences as a family. Based on the reproducibilities of PCR and HTS, we therefore assume that these differences were caused by PCR errors. However, it is not possible to provide a definite answer without replicating the PCR. Further sequencing of the sample using a high-fidelity enzyme confirmed that these were artefacts. The Vidjil web application allows followup and sample replicates to be analyzed. If the four sequences were clustered manually, the Vidjil web application would allow both evolutions to be plotted. It is important to note that, in practice, clustering such sequences in a single clone does not change the result by more than one log. 


\begin{tabular}{llll} 
Patient & \multicolumn{2}{c}{ System } & Samples \\
\hline 009 & TRG & & D0, D115, D154 \\
010 & TRG & & D0, D81, D413, D445, D475 \\
011 & TRG & & D0, D61, D92, D119 \\
012 & & IGH & D0, D37, D100 \\
013 & & IGH & D0, D60, D120, D246, D274 \\
014 & & IGH & D0, D38, D93 \\
063 & TRG & IGH & D0, D48, D90, D263, D308 \\
064 & TRG* & IGH & D0, D47, D403, D615, D711, \\
& & & D741, D794, D837 \\
099 & TRG & & D0, D107 \\
100 & TRG & IGH & D0, D42 \\
101 & & IGH & D0, D44 \\
\hline scale & & IGH & D0 + 4 dilutions
\end{tabular}

Table 1: All patients were sequenced at diagnosis (D0) and at some follow-up time points. The days indicated for follow-up samples are post diagnosis. * Note that TRG in patient 064 was sequenced at five points (D0, D47, D615, D711, D837) with the previous protocol, reported in [30].

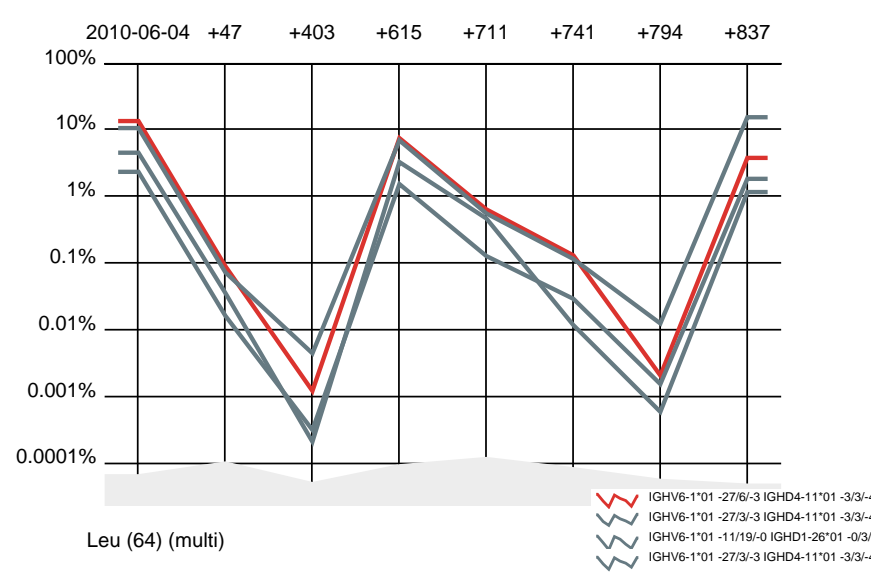

Figure 1: Evolution of the four first IGHV6$1 * 01 /$ IGHD4- $11^{*} 01 /$ IGHJ $4{ }^{*} 02$ clones (raw data, before further clustering, sequences on Figure 3 for patient 064. Compare to Figure 1, bottom right. 
... VVVVVVVNNNNJJJJJJ. . .

$\begin{array}{lrrr}\text { clone } & 10776 & 5.2 \% & \text { TATCAGCAGCCTAAAGGCTGAGGACACCCGACAGGGTATGGACGTCTGGGGCCAAGGGAC } \\ \text { pcr_bias1 } & 4102 & 2.0 \% & \text { TATCAGCAGCCTAAAGGCTGAGGACACCCGACAGGGTATGGACGTCTG---CCAAGGGACCCT } \\ \text { pcr_bias2 } & 3116 & 1.5 \% & \text { ATATCAGCAGCCTAAAGGCTGAGGACACCCGACAGG-TATGGACGTCTG---CCAAGGGACCCT }\end{array}$

Figure 2: Main biological clone (IGHV7-40*03 -22/ACAG/-17 IGHJ6*02) and two PCR artefacts seen in only one PCR replicate (PCR A) in patient 014. No read with the sequence for pcr $\_$bias 1 and pcr_bias 2 was detected in PCR B.

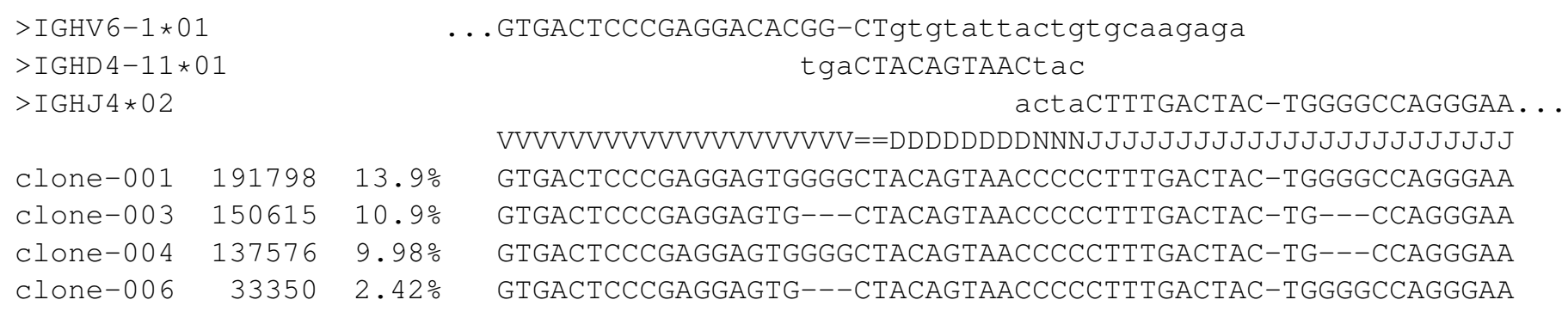

Figure 3: Representative sequences of the largest clones identified as IGHV6-1*01/IGHD4$11^{*} 01 / \mathrm{IGHJ} 4^{*} 02$ in patient 064 , aligned with IMGT/GENE-DB relevant germline genes. For each clone, the number of reads and the proportion among all analyzed reads are given. Together, these clones include 571,221 reads, that is $47.6 \%$ of reads analyzed as IGH (and $36.7 \%$ of all analyzed reads). Two positions, marked by =, can be assigned to either the $\mathrm{V}$ or $\mathrm{D}$ segment. The first four clones show all four possible combinations of the mutation G / GGGG at two different positions: one at the end of the V segment, and another in the middle of the J segment. Further sequencing after PCR using a high-fidelity enzyme showed that clone-001 was the only biological clone, and the others were the result of PCR artefacts.

\begin{tabular}{|c|c|c|c|c|c|c|c|c|c|c|}
\hline \multirow{4}{*}{$\begin{array}{l}\text { TRG 1-10 } \\
\text { (BIOMED-2) }\end{array}$} & Vgf1 & + & $5^{\prime} \mathrm{GGA}$ & AGG & $\mathrm{CCC}$ & CAC & AGC & RTC & TT $3^{\prime}$ & \\
\hline & Vg10 & + & $5^{\prime} \mathrm{AGC}$ & $\mathrm{TG}$ & GGT & $\mathrm{AAG}$ & $\mathrm{ACA}$ & AGC & AA $3^{\prime}$ & \\
\hline & J1J2 & - & $5^{\prime}$ GTG & TTG & TTC & CAC & TGC & $\mathrm{CAA}$ & $\overline{A G A}$ & G 3' \\
\hline & $\mathrm{JP1} / 2$ & - & $5^{\prime}$ TTA & $\mathrm{CCA}$ & GGC & GAA & GTT & $\mathrm{ACT}$ & ATG & AGC $3^{\prime}$ \\
\hline \multirow{3}{*}{$\begin{array}{l}\text { TRG } 9 \\
\text { (BIOMED-2) }\end{array}$} & Vg9 & + & $5^{\prime} \mathrm{CGG}$ & CAC & TGT & $\mathrm{CAG}$ & $\mathrm{AAA}$ & GGA & $\operatorname{ATC}$ & \\
\hline & $\mathrm{J} 1 \mathrm{~J} 2$ & - & $5^{\prime} \mathrm{GTG}$ & TTG & TTC & CAC & TGC & CAA & AGA & G 3' \\
\hline & $\mathrm{JP1} / 2$ & - & $5^{\prime}$ TTA & $\mathrm{CCA}$ & GGC & GAA & GTT & $\mathrm{ACT}$ & ATG & AGC $3^{\prime}$ \\
\hline \multirow[t]{2}{*}{$\operatorname{IgH}$} & FR1 & + & $5^{\prime} \mathrm{AGG}$ & TGC & AGC & TGS & WGS & AGT & $\mathrm{CDG}$ & G 3' \\
\hline & JHDN & - & $5^{\prime} \mathrm{ACC}$ & TGA & GGA & GAC & GGT & GAC & CAG & GGT 3' \\
\hline
\end{tabular}

Table 2: Forward (+) and reverse (-) primers used for TRG and IGH PCR. 